\title{
Planetary Torques as the Viscosity of Protoplanetary Disks
}

\author{
J. Goodman and R. R. Rafikov \\ Princeton University Observatory, Princeton, NJ 08544 \\ jeremy@astro.princeton.edu
}

\begin{abstract}
We revisit the idea that density-wave wakes of planets drive accretion in protostellar disks. The effects of many small planets can be represented as a viscosity if the wakes damp locally, but the viscosity is proportional to the damping length. Damping occurs mainly by shocks even for earth-mass planets. The excitation of the wake follows from standard linear theory including the torque cutoff. We use this as input to an approximate but quantitative nonlinear theory based on Burger's equation for the subsequent propagation and shock. Shock damping is indeed local but weakly so. If all metals in a minimum-mass solar nebula are invested in planets of a few earth masses each, dimensionless viscosities [alpha] of order $\operatorname{dex}(-4)$ to $\operatorname{dex}(-3)$ result. We compare this with observational constraints. Such small planets would have escaped detection in radial-velocity surveys and could be ubiquitous. If so, then the similarity of the observed lifetime of $\mathrm{T}$ Tauri disks to the theoretical timescale for assembling a rocky planet may be fate rather than coincidence.
\end{abstract}

Subject headings: planets and satellites: general — solar system: formation — (stars:) planetary systems

\section{Introduction}

The disks of young low-mass protostars ("protostellar disks") are observed to accrete at rates $\sim 10^{-8} M_{\odot} \mathrm{yr}^{-1}$ (Hartmann et al. 1998). A comparable average accretion rate is implied by the disk masses inferred from dust emission $\left(\sim 10^{-2 \pm 1} M_{\odot}\right.$, Osterloh \& Beckwith 1995) when combined with the maximum ages of $\mathrm{T}$ Tauri stars that show evidence for disks $\left(\sim 10^{6-7} \mathrm{yr}\right.$, Strom et al. 1989), or with radiochemical estimates for the lifetime of the protostellar nebula (Podosek \& Cassen 1994). Indeed, the evidence that young protostars are surrounded by viscous accretion disks has only strengthened in the quarter century since Lynden-Bell \& Pringle (1974) proposed it.

While the effective viscosity of the hotter accretion disks surrounding degenerate stars and black holes is probably due to magnetohydrodynamic (MHD) turbulence (cf. Balbus \& Hawley 1998), protoplanetary disks may be too cold, too weakly ionized, and too resistive to sustain MHD turbulence (Blaes \& Balbus 1994; Jin 1996; Hawley \& Stone 1998) except perhaps at small radii, 
in surface layers (Gammie 1996), or during FU Orionis-type outbursts (Hartmann et al. 1993). In view of several physical uncertainties in the ionization balance (cf. Gammie 1996), and the possibly subtle nature of the conductivity itself (Wardle 1999), this conclusion is only tentative. But it is probably fair to say that the best reason for supposing that the viscosity of protoplanetary disks is magnetic remains the lack of a theoretically attractive alternative. Convection (Lin \& Papaloizou 1980) and nonlinear shear instability (Zahn 1991; Dubrulle 1992) have been proposed, but these mechanisms appear to fail because of the very strongly stabilizing influence of a positive radial gradient in specific angular momentum (Ryu \& Goodman 1992; Stone \& Balbus 1996; Balbus et al. 1996).

Larson (1989) suggested that density waves might provide the efffective viscosity mechanism in protostellar disks. As he made clear, wave transport is rather different from viscous transport. In the standard theory of thin accretion diks, viscosity $(\nu)$ plays two roles. Firstly, it provides an outward angular-momentum flux $\left(F_{J}\right)$, a torque exerted by the part of the disk interior to a given radius on the part exterior. Secondly, it heats the disk by dissipating the differential rotation. In steady state, $F_{J}$ balances the inward flux of angular momentum carried by the accreting matter, while the dissipation balances the loss of its orbital energy. Even if the disk is not steady, provided only that it is truly viscous, then

$$
F_{J}=-\nu \times 2 \pi \Sigma r^{3} \frac{d \Omega}{d r}
$$

where $\Sigma$ is the surface density and $\Omega$ the angular velocity. Also, the dissipation rate per unit area is related to $F_{J}$ by

$$
\dot{E}=-F_{J} \frac{d \Omega}{d r} .
$$

Any dissipative mechanism such that $F_{J} \propto d \Omega / d r$ and eq. (1) hold can be described by an effective viscosity $\nu_{\text {eff }}$; one expects this to be the case for dissipative mechanisms that are sufficiently local.

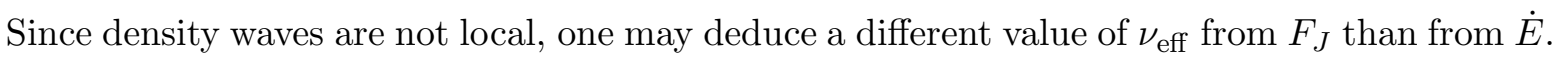
In particular, inviscid linear theory (Goldreich \& Tremaine 1980, henceforth GT80) predicts that a planet embedded in a gas disk excites density waves at the inner and outer Lindblad resonances of its (circular) orbit; these waves carry $F_{J}>0$ but $\dot{E}=0$ by assumption, so that $\nu_{\dot{E}}=0$ but $\nu_{J}>0$. Actually, the theory implicitly assumes some damping at large distance from the resonances, else the waves would reflect from the edges of the disk and produce zero net flux. If one is concerned with the torques on the disk only, then the damping length is unimportant as long as it is large. But without dissipation, the waves cause no secular changes to the orbits of the gas even at the Lindblad resonances (Goldreich \& Nicholson 1989).

By demanding that eq. (1) be satisfied, Larson (1990) found that steady spiral shocks produce

$$
\alpha \sim 0.013\left[(c / V)^{3}+0.08(c / V)^{2}\right]^{1 / 2}
$$

independently of the source of excitation of the waves. Here $c$ is the sound speed and $V=r \Omega$ the orbital velocity. For $c / V=0.03$, perhaps characteristic of the later phases of the protosolar nebula 
at $1 \mathrm{AU}$, this gives $\alpha \approx 10^{-4}$. Larson's result depends upon a number of additional assumptions, some inspired by earlier work by Spruit (1987) on self-similar shocks: the waves were taken to form two spiral arms separated by $180^{\circ}$ in azimuth; and because they would be very tightly wrapped since $c \ll V$, Larson (1990) approximated the wave profile by analyzing a train of perfectly axisymmetric nonlinear waves. Most importantly, at least by contrast with our own work reported below, no source of excitation for the waves exists within the range of radii where eq. (2) applies. Larson argued, however, that a bisymmetric spiral shock of the required strength could be launched from the $2: 1$ resonance of a jovian-mass planet, and he deduced an accretion timescale $\sim 10^{6-7} \mathrm{yr}$ in accord with observational requirements.

Jupiter-mass planets have been discovered during the past few years by exquisitely sensitive radial-velocity surveys (Marcy et al. 2000). Most of these planets have orbital periods shorter than that of Mercury. They are detected around $4-5 \%$ of the stars surveyed, mostly G, F, and K dwarfs. There are indications that stars of higher-than-solar metallicity are preferred (Gonzalez 1997; Santos et al. 2000; Gonzalez et al. 2000). Because of observational selection effects, it is probably too early to derive the incidence of giant planets in long-period orbits $(\sim 10 \mathrm{yr})$. If such planets are as rare as those on short-period orbits, however, then we must seek another explanation for the observed accretion rates and inferred lifetimes of most protostellar disks. Nevertheless, it remains an intriguing notion that planet formation is not just constrained by disk lifetimes, but may actually determine them.

Our paper therefore inquires whether accretion could be driven by planets of roughly terrestrial mass, which radial-velocity surveys would not have detected. Other things being equal, the torque exerted on the disk by a planet is proportional to the square of the planet's mass $\left(M_{p}\right)$, so the influence of $M_{p} \sim 10^{-5} M_{\odot}$ might be thought negligible compared with that of $M_{p} \sim 10^{-3} M_{\odot}$. Just because its local effects are so strong, however, the larger planet will almost certainly open a gap in the disk, whereas the smaller one will not; since the torque per unit radius (smoothed over resonances) decreases rapidly with distance from the planet (GT80), the shorter range of its interaction may partly compensate for the terrestrial planet's smaller mass. At solar abundance there could be tens of earth-mass planets in the disk, each too small to have captured a significantly massive atmosphere. We assume that the planets are distributed through the disk in proportion to the local surface density of the gas, neglecting orbital migration and radial changes in the timescale of planet formation. Unlike Larson, therefore, we have a distributed source of excitation for density waves, so that eq. (2) need not apply (although in fact we obtain a comparable value of $\alpha$ in the minimum-mass solar nebula).

As discussed, no sensible effective viscosity can be derived for density waves without considering their damping. $\S 2$ shows for a generic damping mechanism in a thin disk that if the waves are absorbed over a distance small compared to the disk radius, but possibly large compared to its thickness, and if the local damping rate depends mainly on distance from the planet, then eq. (1) is satisfied so that a consistent value of $\nu_{\text {eff }}$ or $\alpha$ can in fact be defined. However, the viscosity is directly proportional to the damping length. After briefly considering other mechanisms in $\S 3$, we 
conclude that shock damping is probably most important even at $M_{p} \sim M_{\oplus}$. In $\S 4$, we calculate the excitation of the wake from linear theory, which is valid for sufficiently small $M_{p}$ [eq. (31]. In $\S 5$ and the Appendix, we show that the subsequent nonlinear propagation of the wake away from the planet can be reduced to Burger's equation using some transformations and some approximations that should be accurate for sufficiently low-mass planets in sufficiently thin disks. We describe quantitatively the formation of the shock and its subsequent decay with increasing distance from the planet. Most importantly, we obtain formulae for the shock-damping length, and hence for $\alpha_{\text {eff }}$, as functions of $M_{p}$ and local disk parameters. $\S 6$ summarizes our main results and discusses their implications for disks resembling a standard minimum-mass solar nebula.

\section{Effective $\alpha$-parameter.}

Let $f_{J}^{(0)}\left(M_{p}, r_{p}\right)$ be the angular-momentum flux excited by a planet of small mass $M_{p}$ and orbital radius $r_{p}$, embedded in a disk of local surface density $\Sigma$, sound speed $c$, and angular velocity $\Omega(r) \approx\left(G M_{*} / r^{3}\right)^{1 / 2}$, where $M_{*}$ is the mass of the central star. According to GT80,

$$
f_{J}^{(0)}\left(M_{p}, r_{p}\right)=\left(G M_{p}\right)^{2} \frac{\Sigma r_{p} \Omega}{c^{3}} \times\left\{\frac{4}{9} \mu_{\max }^{3}(Q)\left[2 K_{0}(2 / 3)+K_{1}(2 / 3)\right]^{2}\right\} .
$$

The dimensionless quantity $\mu$ is defined in such a way that $\mu \Omega r / c \gg 1$ is roughly the largest azimuthal harmonic contributing to the flux (the "torque cutoff"); it is a function of the gravitational stability parameter $Q=c \Omega / \pi G \Sigma$ (Toomre 1964). Selfgravity is probably very weak in the later evolutionary phases of protostellar disks, so it is appropriate to take $Q \rightarrow \infty$. In this limit, it follows from GT80's results that the quantity in curly brackets $\approx 0.93$. As acknowledged by GT80 and elaborated by later authors, eq. (3) is valid only to leading order in $c / \Omega r$, the ratio of disk thickness to radius. At the next order, there is a slight imbalance between the interior $\left(r<r_{p}\right)$ and exterior $\left(r>r_{p}\right)$ fluxes; the difference between the two torques represents a net torque on the planet, whose orbit therefore "migrates," probably towards smaller $r_{p}$ (Ward 1997, and references therein). The effective viscosity we calculate depends on the sum of the interior and exterior fluxes, so we neglect the difference between them.

The flux rises quickly to a constant value (3) over a radial distance from the planet of order $l \equiv c /|r d \Omega / d r|^{-1}$, the length over which the rotation velocity changes by Mach 1 . The strongest Lindblad resonances giving rise to the wake lie within this distance. Absent damping, the angular momentum flux is radially constant at $\left|r-r_{p}\right| \gg l$. We can characterize the strength of damping by the reciprocal of the damping length $l_{d}$ at which $f_{J}$ falls to $\sim e^{-1}$ of eq. (3).

The larger the damping length, the greater the dissipation of orbital energy by the densitywave wake, for the following reason. If a quantity $\Delta J$ of angular momentum is added to the disk in the form of waves having angular pattern speed $\Omega_{p}$ (in steady state, the wake has the pattern speed of the planet), the work required to create the waves is $\Omega_{p} \Delta J$. When these waves damp at radius $r$, the work done on the mean flow there is $\Omega(r) \Delta J$. The difference $\left[\Omega_{p}-\Omega(r)\right] \Delta J$ represents 
the energy available to be dissipated, assuming that this is positive. It can be shown that $\Delta J$ is positive (negative) for waves excited exterior (interior) to the planet in the approximation that $\Omega_{p}=\Omega\left(r_{p}\right)$. From these considerations, it follows that the total mechanical energy dissipated by the wake of the one planet is

$$
\dot{e}\left(M_{p}, r_{p}\right)=\int_{0}^{\infty}\left[\Omega(r)-\Omega_{p}\right] \frac{d f_{J}}{d r} d r
$$

Clearly $\dot{e} \propto l_{d}$, asssuming $l_{d} \ll r_{p}$. Suppose that the background properties of the disk are approximately constant over radial distances of order $l_{d}$. Write the angular momentum flux in terms of its ideal asymptotic value (3) and a dimensionless distribution function, $f_{J}(r)=f_{J}^{(0)} \cdot \phi\left(r-r_{p}\right)$. Our notation presumes a damping mechanism such that $\phi$ depends much more rapidly on $r-r_{p}$ than on $\left(r+r_{p}\right) / 2$, the latter dependence being significant only on the scale of $r$ itself. The integral (4) can now be approximated by

$$
\dot{e}\left(M_{p}, r_{p}\right)=f_{J}^{(0)}\left(M_{p}, r_{p}\right)\left(\frac{d \Omega}{d r}\right)_{r_{p}} \int_{-\infty}^{\infty} x \frac{d \phi}{d x} d x
$$

where $x \equiv r-r_{p}$. The lower limit of integration has been extended to $-\infty$ for convenience since $\phi(x) \approx 0$ unless $|x| \ll r_{p}$.

Now suppose that there are many planets of the same mass distributed through the disk in numbers $N(r)$ per unit radius, and that $r N(r) \gg 1$. Then the total flux at radius $r$ is (on average)

$$
F_{J}(r)=\int_{0}^{\infty} N\left(r_{p}\right) f_{J}^{(0)}\left(M_{p}, r_{p}\right) \phi\left(r-r_{p}\right) d r_{p} \approx N(r) f_{J}^{(0)}\left(M_{p}, r\right) \int_{-\infty}^{\infty} \phi(x) d x,
$$

assuming that $N(r)$ and $f_{J}^{(0)}\left(M_{p}, r\right)$ vary only slowly with radius. Similarly, the dissipation rate per unit radius at $r$ is

$$
\begin{aligned}
\dot{E}(r) & =\int_{0}^{\infty}\left[\Omega(r)-\Omega\left(r_{p}\right)\right] N\left(r_{p}\right) f_{J}^{(0)}\left(M_{p}, r_{p}\right) \frac{\partial \phi}{\partial x}\left(r-r_{p}\right) d r_{p} \\
& \approx \frac{d \Omega}{d r} N(r) f_{J}^{(0)}\left(M_{p}, r\right) \int_{-\infty}^{\infty} x \frac{d \phi}{d x} d x=-\frac{d \Omega}{d r} F_{J}(r),
\end{aligned}
$$

where the last step has used integration by parts. So, as promised in $\S 1$, equation (1) is satisfied by density-wave wakes under the assumptions of many planets and local damping.

In the standard theory of viscous accretion disks (Pringle 1982), the viscous energy dissipation rate per unit radius is related to the Shakura \& Sunyaev (1973) viscosity parameter $\alpha$ by (assuming $\left.\Omega \propto r^{-3 / 2}\right)$

$$
\dot{E}_{\mathrm{visc}}=\alpha \frac{9 \pi}{2} r \Omega \Sigma c^{2}
$$


Let the planets have average mass $\left\langle M_{p}\right\rangle$ and let them account for a fraction $Z_{p} \ll 1$ of the total nebular mass; we expect that $Z_{p}$ is comparable to the metallicity of the gas if planet-formation is efficient but the planets are too small to have captured massive gaseous envelopes (Mizuno et al. 1978; Podolak et al. 1993; Ikoma et al. 2000) Then the average number of planets per unit radius is

$$
N=\frac{2 \pi r Z_{p} \Sigma}{\left\langle M_{p}\right\rangle}
$$

The total dissipation rate per unit radius due to planetary wakes is $N\langle\dot{e}\rangle$, so by comparison with eq. (8),

$$
\alpha_{\mathrm{eff}}=\frac{4}{9} \frac{Z_{p}}{\Omega c^{2}} \frac{\langle\dot{e}\rangle}{\left\langle M_{p}\right\rangle} .
$$

Despite the relations (3) and (5), we are not yet in a position to evaluate $\alpha_{\text {eff }}$ numerically because we have not determined the width of the distribution $\phi$, or equivalently, the damping length.

In the rest of the paper we assume a minimum mass solar nebula MMSN, cf. Hayashi 1981:

$$
\begin{gathered}
\Sigma(r)=1700 \mathrm{~g} \mathrm{~cm}^{-3} r_{A U}^{-3 / 2}, \\
c(r)=1.2 \mathrm{~km} \mathrm{~s}^{-1} r_{A U}^{-1 / 4},
\end{gathered}
$$

where $r_{A U}$ is the radius $r$ expressed in astronomical units. For these parameters,

$$
Q=67 r_{A U}^{-1 / 4}
$$

which is effectively infinite for our purposes, so that we completely neglect self-gravity of the disk. The MMSN plausibly corresponds to the state of protostellar disks as observed around T Tauri stars, but disks are likely to have been more massive at earlier evolutionary phases when the star was enshrouded by infalling dust and gas. Self-gravity could then have been important to the effective viscosity and accretion rate (Gammie 2000).

Another important assumption is that the sound speed is independent of height above the midplane. This is appropriate for the later evolutionary phases of protostellar disks, when the local thermal structure is probably dominated by passive reprocessing of radiation from the central star (Chiang \& Goldreich 1997), and it is in these phases that planets are most likely to be dynamically important. Vertical isothermality allows us to treat even short-wavelength acoustic disturbances two-dimensionally, i.e. in radius and azimuth. When the disk is prodominantly self-luminous, as is likely in earlier phases and during FU Orionis outbursts, the temperature may decrease away from the midplane, in which case acoustic waves will concentrate toward the surface and perhaps damp more quickly than we estimate here (Lubow \& Ogilvie 1998; Ogilvie \& Lubow 1999). The thermal stratification of an active disk is very uncertain, however, since the distribution of dissipation over height is poorly understood; in some models, dissipation occurs mainly near the surface, which would also tend to make the disk vertically isothermal (Gammie 1996). 


\section{Linear damping}

Linear damping mechanisms are those for which the damping length is independent of amplitude and which do not couple different azimuthal harmonics of the wake. One possibility is a background viscosity $\nu_{0}$ that acts on density waves as well as the mean shear. Takeuchi et al. (1996) showed that in this case the wave's angular momentum decays with distance as (for definiteness, consider $\left.r>r_{p}\right) f_{J, m} \propto \exp \left[-2 \int^{r} \Delta k_{\nu} d r\right]$, where $f_{J, m}$ is the angular momentum flux carried by the $m^{\text {th }}$ azimuthal harmonic, and $\Delta k_{\nu}$ is the contribution to the imaginary part of the WKBJ wavenumber due to viscosity:

$$
\operatorname{Re}(k)^{2} \approx \frac{m^{2}\left(\Omega-\Omega_{p}\right)^{2}-\kappa^{2}}{c^{2}}
$$

and

$$
\Delta k_{I} \approx \nu_{0}\left[\frac{4}{3}+\frac{\kappa^{2}}{m^{2}\left(\Omega-\Omega_{p}\right)^{2}}\right] \frac{m\left(\Omega_{p}-\Omega\right)}{2 c^{2}} \operatorname{Re}(k),
$$

The most important contributions to the flux are for $m \sim \mu$, where $\mu \sim r / l \gg 1$ is the torque cutoff $(\mathrm{GT} 80)$, so the terms involving the epicyclic frequency $\kappa(\approx \Omega)$ can be neglected beyond a few $l$ from the planet. Hence $\Delta k_{I} \sim\left(r-r_{p}\right)^{2} / l^{4}$, so

$$
f_{J, m} \sim f_{J, m}^{(0)} \exp \left[-\alpha_{0}\left(r-r_{p}\right)^{3} / h^{3}\right] .
$$

Here $\alpha_{0} \equiv \nu_{0} / \Omega h^{2}$ and $h \equiv c / \Omega r=(3 / 2) l$ is the disk thickness. Hence the damping length is $l_{d} \sim \alpha_{0}^{-1 / 3} h$. If $\alpha_{0}$ is as large as $10^{-3}$, then there is no need to invoke density waves to explain accretion. On the other hand, if $\alpha_{0} \ll 10^{-3}$ then $l_{d} \gg 10 h$.

Cassen \& Woolum (1996) have proposed another linear damping mechanism: radiative losses from the disk surface. They conclude that the effect is strong, but we believe that they made inappropriate use of the adiabatic approximation. Cassen \& Woolum estimate the radiative losses by evaluating the adiabatic (lossless) density-wave eigenfunction at the disk photosphere. Because they take an isothermal background state, as appropriate for a passive disk warmed by the central star, the first-order temperature perturbation at the photosphere is as large as it is at the disk midplane, so that their damping rate is almost independent of the total optical depth of the disk (cf. their Fig. 1). This result is implausible. By contrast, we find that if the non-adiabatic effects are included self-consistently in the linear analysis, then the damping rate is inversely proportional to the total optical depth and is very much reduced compared to their estimate. The typical optical depths in the MMSN are $\tau \sim 10^{3}-10^{4}$.

For the planetary wake, radiative diffusion in the radial direction [neglected by Cassen \& Woolum] is much more important than losses from the surface, because the radial wavelength is typically shorter than the vertical thickness. The process is similar to damping of a sound wave in air by thermal conduction. Adapting formulae from Landau \& Lifshitz (1959), we find

$$
\Delta k_{I} \sim \frac{k^{2} h^{2}}{c} \frac{2 m_{H} \sigma_{B} T^{3}}{\Sigma k_{B} \tau},
$$


where $\sigma_{B}$ is the Stephan-Boltzmann constant, $2 m_{H}$ is the mass of the hydrogen molecule, and $k_{B}$ is Boltzmann's constant (and $c$ is still the speed of sound, not of light). Using eq. (14) for $k$, we find that the damping length due to radiative diffusion is

$$
l_{d} \sim h\left(\frac{\Omega \Sigma k_{B} \tau}{2 m_{H} \sigma_{B} T^{3}}\right)^{1 / 3} .
$$

The most interesting feature of this expression is that now $l_{d} \propto \tau^{1 / 3}$, so that it is much smaller than in the case of radiative losses from surface where (in our analysis) $l_{d} \propto \tau$. Numerical estimates show, however, that the damping length is still $\gtrsim r$ for typical conditions.

We conclude that the obvious linear damping mechanisms produce damping lengths as large as the radius itself, or larger. Therefore, these mechanisms are unimportant compared to shock damping which, as will be seen, leads to a damping length that scales with planetary mass as $\propto M_{p}^{-2 / 5}$ but is still only a modest multiple of the disk thickness even if $M_{p}$ is as small as $M_{\oplus}$.

\section{Linear excitation of a planetary wake}

Even if the planet does not open a gap in the disk, there is a minimum radial separation $\left|r-r_{p}\right|=l$ at which density waves can be excited because at smaller distances the velocity of the gas in the corotating frame of the planet is subsonic, and a stationary perturber does not excite acoustic waves in a subsonic flow (Landau \& Lifshitz 1959). This explains the torque cutoff (GT80), at least in nonselfgravitating disk where the density waves are basically acoustic. For a planet of sufficiently low mass [eq. (31)], the forces exerted at this minimum distance are weak enough so that the excitation of the wake and its angular momentum flux can be accurately calculated by linear theory. It is well known that in the absence of linear damping (viscosity, thermal conductivity), a sound wave of small but finite amplitude propagating into still air eventually shocks; the distance to the shock is proportional to the wavelength and inversely proportional to the initial amplitude (Landau \& Lifshitz 1959; Whitham 1974). In a disk the shock length is shorter and the dependence on initial initial amplitude is weaker because differential rotation compresses the radial width of the wake as it propagates.

Linear excitation has been studied by Goldreich \& Tremaine (1978, henceforth GT78), GT80, Artymowicz (1993), Ward (1997), and others. The relative phases of the azimuthal Fourier harmonics of the wake are normally discarded because they are not relevant to the torque. But the onset of the shock depends upon the local slope of the wake front, which in turn depends upon these phases. Therefore, we have repeated the linear calculation using the methods of GT78 and GT80.

As usual, $x=r-r_{p}$ and $y=r_{p}\left(\theta-\theta_{p}\right)$, denote pseudo-cartesian radial and azimuthal coordinates in a corotating system centered on the planet, and all properties of the background flow are expanded to lowest order in $x / r_{p}$. The background surface density $\Sigma$ and sound speed $c$ 
are made constant, as are the rotation rate (=Coriolis parameter) $\Omega$, shear rate $2 A \equiv r d \Omega / d r$, and vorticity $2 B \equiv 2(\Omega+A)$. The background flow with respect to the planet is $2 A x \mathbf{e}_{y}$. No distinction is made between $\Omega$ and the angular velocity of the planet, since this is not important for the angular momentum flux carried by the wake to leading order in $h / r_{p}$ [but vital to the net torque on the planet (Ward (1997)]. Although we are interested in keplerian disks, for which $2 A / \Omega=-3 / 2$ and $2 B / \Omega=+1 / 3$, the physics is clarified by retaining $A$ and $B$ in basic formulae. We use the Mach- 1 distance $l \equiv c /|2 A|$ rather than $h=c / \Omega=(3 / 2) l$ as a reference length. A convenient unit for the planetary mass is

$$
M_{1} \equiv \frac{c^{3}}{|2 A| G},
$$

which reduces to $2 c^{3} / 3 \Omega G$ in a keplerian disk. In linear theory, the amplitude of the wake is $\propto M_{p}$, so it is sufficient to calculate as if $M_{p}=M_{1}$ and scale the results accordingly. Note that $M_{p} \gtrsim M_{1}$ is also the point at which the linear approximation begins to fail; the Roche lobe of the planet becomes $\gtrsim l$, and a gap may open in the disk $(\S 6)$.

The wake is steady in the planet frame, so that its perturbations in radial and azimuthal velocity, $u, v \ll c$, and the relative perturbation in surface density, $\sigma=\delta \Sigma / \Sigma \ll 1$, are functions of $(x, y)$ only. Their spatial Fourier transforms $\hat{u}, \hat{v}$, and $\hat{\sigma}\left(k_{x}, k_{y}\right)$ satisfy (GT78, GT80)

$$
\begin{aligned}
\frac{d^{2} \hat{v}}{d \tau^{2}} & +\left[c^{2} k(\tau)^{2}+\kappa^{2}\right] \hat{v}=-i k_{y} \frac{d \hat{\phi}}{d \tau}+2 i k_{x}(\tau) B \hat{\phi} \\
\hat{u} & =-\frac{1}{c^{2} k_{y}^{2}+4 B^{2}}\left(2 B \frac{d \hat{v}}{d \tau}-c^{2} k_{x} k_{y} \hat{v}+2 B i k_{y} \hat{\phi}\right), \\
\hat{\sigma} & =\frac{i}{c^{2} k_{y}^{2}+4 B^{2}}\left(k_{y} \frac{d \hat{v}}{d \tau}+2 B k_{x} \hat{v}+i k_{y}^{2} \hat{\phi}\right) .
\end{aligned}
$$

The timelike variable $\tau$ is related to the pitch angle of the wave:

$$
\tau \equiv-\frac{k_{x}}{2 A k_{y}}
$$

the notation is standard and will not be confused with optical depth. An individual Fourier component evolves at constant $k_{y}$ but varying $k_{x}$ due to the differential rotation. Since $A<0$, all Fourier information flows along the characteristics $d k_{x} / d \tau=-2 A k_{y}$ from leading $(\tau<0)$ to trailing $(\tau>0)$ waves, and eqs. (20) describe the evolution of the amplitudes along those characteristics. The quantity $k$ is the instantaneous wavenumber $\sqrt{k_{x}^{2}+k_{y}^{2}}$, and $\hat{\phi}=-2 \pi G M_{p} / k$ is the Fourier transform of the point-mass planetary potential. Appropriate initial conditions are $\hat{v}=0$ at $\tau=-\infty$. Unlike GT80, we have neglected the selfgravity of the disk.

Unless otherwise noted, the rest of this section assumes units $c=-2 A=1$, so that $l=1$, and the wake amplitude is scaled to $M_{p}=M_{1}$.

We solved eqs. (20) numerically to obtain $(\hat{u}, \hat{v}, \hat{\sigma})$ on a grid of $N_{x} \times N_{y}=4096 \times 8192$ points in $\left(k_{x}, k_{y}\right)$. The very large grid proved necessary to minimize numerical artifacts of the discrete 
Fourier transform (DFT). We chose the maximum (Nyquist) value of $k_{y}=8$, well beyond the torque cutoff. The corresponding azimuthal grid resolution and periodicity length are $\Delta y=(\pi / 8)$ and $N_{y} \Delta y \approx 3217$, respectively. Since the locus of the wake is approximately $y \approx-x^{2} \operatorname{sign}(x) / 2$, we chose $L_{x}=2 \sqrt{L_{y}} \approx 113$, implying a radial resolution $\Delta x=L_{x} / N_{x}=0.028$.

The Fourier data were filtered in pitch angle to avoid aliasing. When $k_{x} \gg k_{y} \& \kappa$, the homogeneous solutions to eqs. (20) change phase by $k_{x} \Delta \tau=\tau \Delta k_{x}$ from $k_{x}$ to $k_{x}+\Delta k_{x}$, where $\Delta k_{x}=2 \pi / L_{x}$ is the resolution in Fourier space, and $k_{x}=\tau k_{y}$ in the present units. This phase change should be $<\pi$, in other words $\tau_{\max }=L_{x} / 2 \approx 57$, else the phase will be aliased and the inverse DFT will assign the wave to the wrong $x$ position. We multiplied the Fourier components by the pitch-angle filter

$$
W\left(k_{x}, k_{y}\right)= \begin{cases}1 & \text { if } \tau<\tau_{\max } / 2 \\ 2\left(1-\tau / \tau_{\max }\right) & \tau_{\max } / 2 \leq \tau<\tau_{\max } \\ 0 & \tau \geq \tau_{\max }\end{cases}
$$

Because the pitch angle increases linearly with $|x|$ in coordinate space, this filter attenuates the wake at $|x| \gtrsim L_{x} / 4$. We also used a similar filter in the $k_{y}$ direction, tapering linearly from unity at $\left|k_{y}\right|=k_{y, \text { Nyquist }} / 2$ to zero at $\left|k_{y}\right|=k_{y, \text { Nyquist }}$.

We checked our calculation against the angular momentum flux reported by GT80 in two different ways. One can show that as $L_{x}, L_{y} \rightarrow \infty$,

$$
f_{J}=\lim _{\tau \rightarrow \infty} \frac{r_{p} \Sigma}{4 \pi^{2} l} \int_{0}^{\infty}\left\langle\hat{u}\left(k_{x}, k_{y}\right) \hat{v}^{*}\left(k_{x}, k_{y}\right)\right\rangle_{\tau}\left|k_{y}\right| d k_{y},
$$

the average being taken over oscillations in $\tau$ at fixed $k_{y}$. We evaluated a numerical approximation to this formula in Fourier space (before applying the windows discussed above) and found agreement with the flux (3) reported by GT80 for $Q=\infty$ to the accuracy they quoted, viz. two significant digits. We also evaluated the flux as a function of $x$ in coordinate space using eq. (32). The errors are larger because the coordinate-space wake is softened by the pitch-angle and $k_{y}$ filters, but nevertheless $f_{J}(x)$ is nearly constant and correct within $\pm 3 \%$ between $|x|=6$ and $|x|=28$.

Fig. 1 shows that beyond a few Mach lengths $(l)$ from the planet, the linearized wake profile is approximately constant as a function of $y+x^{2} \operatorname{sign}(x) / 2$, as expected if the source terms are negligible at $|x| \gg 1$ and the wake propagates as a tightly-wrapped acoustic disturbance (see below). To the extent that this is true, the profiles can be regarded equally well as azimuthal cuts across the wake at fixed radius, or as radial cuts at fixed azimuth but with $x^{2} / 2$ rather than $x$ as radial coordinate. If plotted against $x$, the profile would shrink in width $\propto|x|^{-1}$ for $|x| \gg 1$. The profile is about as compact as possible given the torque cutoff [the integrand of eq. (21) peaks at $k_{y} \approx 0.36 l^{-1}$, showing that the phases of the Fourier components of the wake are closely aligned. 


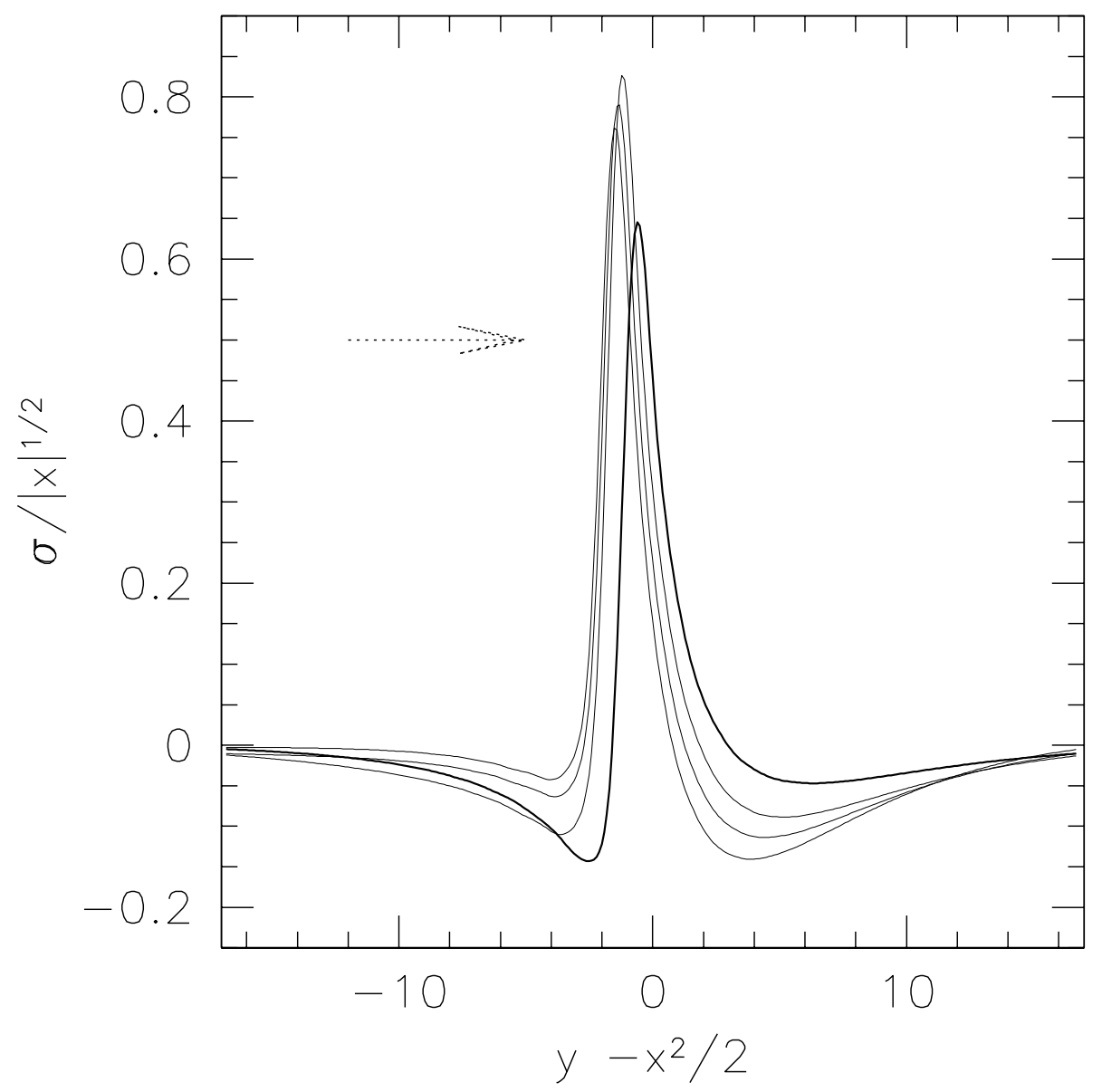

Fig. 1. - Surface-density profile of the linearized wake at $x=-2$ (heavy line), $-4,-6,-8$. Division by $|x|^{1 / 2}$ removes growth due to flux conservation. Arrow shows direction of gas flow relative to profile. See text for dimensional units. 


\section{Nonlinear propagation}

The fully nonlinear steady-state fluid equations in $x y$ are

$$
\begin{aligned}
(2 A x+v) \partial_{y} u+u \partial_{x} u-2 \Omega v+\frac{c^{2}}{\Sigma} \partial_{x} \Sigma & =0 \\
(2 A x+v) \partial_{y} v+u \partial_{x} v+2 B u+\frac{c^{2}}{\Sigma} \partial_{y} \Sigma & =0 \\
(2 A x+v) \partial_{y} \Sigma+u \partial_{x} \Sigma+\Sigma \partial_{x} u+\Sigma \partial_{y} v & =0 .
\end{aligned}
$$

Henceforth $\Sigma$ denotes the full surface density, and $\Sigma_{0}$ its unperturbed value. The sound speed follows the adiabatic law $c^{2}(\Sigma)=c_{0}^{2}\left(\Sigma / \Sigma_{0}\right)^{\gamma-1}$, a good approximation for weak shocks. We have dropped the planetary source terms, since at distances $\gg l$ they are unimportant.

The system (22) is hyperbolic, and in the Appendix, we demonstrate that for $|x| \gg l$, it can be reduced to a single first-order nonlinear equation:

$$
\partial_{t} \chi-\chi \partial_{\eta} \chi=0
$$

which is the inviscid Burger's equation (Whitham 1974). The dimensionless variables appearing here are related to radius, azimuth, and density contrast as follows:

$$
\begin{aligned}
t & \equiv \frac{4}{5}\left|\frac{y}{l}\right|^{5 / 4} \approx \frac{2^{3 / 4}}{5}\left|\frac{x}{l}\right|^{5 / 2}, \\
\eta & \equiv \frac{y}{l}+\frac{x^{2}}{2 l^{2}} \operatorname{sign}(x), \\
\chi & \equiv\left|\frac{y}{l}\right|^{-1 / 4} \frac{\gamma+1}{\gamma-1} \cdot \frac{c-c_{0}}{c_{0}} \approx\left|\frac{y}{l}\right|^{-1 / 4} \frac{\gamma+1}{2} \cdot \frac{\Sigma-\Sigma_{0}}{\Sigma_{0}} .
\end{aligned}
$$

\subsection{The shock}

For almost any smooth choice of initial conditions in Burger's equation (23), the solution will eventually threaten to become double-valued, so that one must fit in a shock (Whitham 1974). The identity $\left(\partial_{t} \chi\right)_{\eta} /\left(\partial_{\eta} \chi\right)_{t}=-\left(\partial_{t} \eta\right)_{\chi}$ converts eq. (23) to the linear equation

$$
\left(\frac{\partial \eta}{\partial t}\right)_{\chi}=-\chi
$$

which says that each point on the wave profile moves forward at a "speed" determined by the value of $\chi$ at that point. A shock develops when higher (larger $\chi$ ) parts of the profile overtake lower ones. The first shock develops where the initial profile is steepest, after a delay

$$
t_{\text {shock }}-t_{0}=\left[\max \left(\frac{\partial \chi}{\partial \eta} \operatorname{sign} \chi\right)\right]_{0}^{-1} .
$$




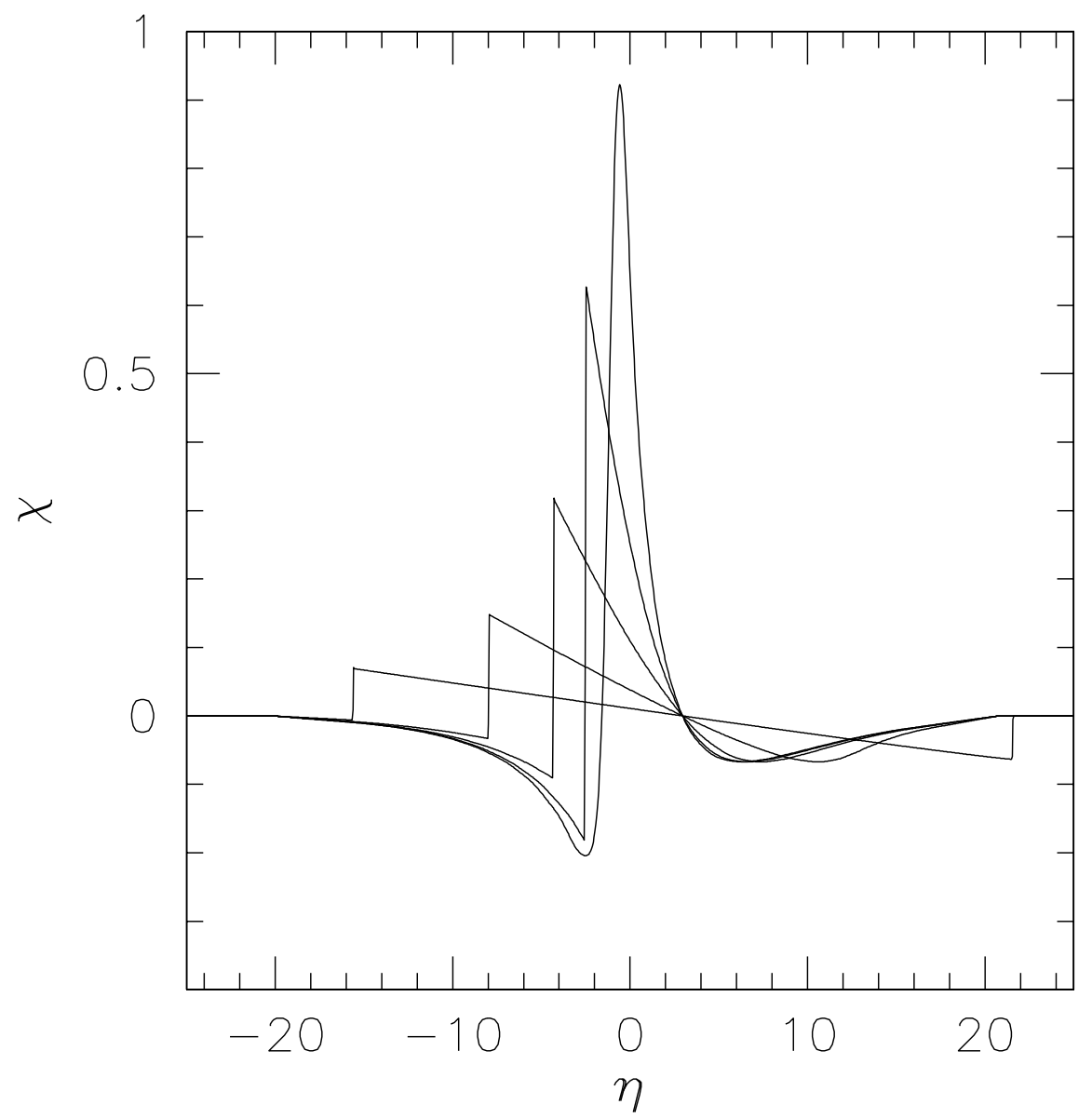

Fig. 2. - Nonlinear evolution of the wake profile according to eqs. (23)-(26), integrated via secondorder advection scheme (van Leer 1977). Initial conditions taken from linear wake profile at $x_{0}=$ $-2 l(t=1.903)$ scaled to planet mass $M_{p}=M_{1}$. Profiles shown at $t-t_{0}=0,4,16,64,256$ (highest to lowest). The latest profile has the expected $\mathrm{N}$-wave shape. 
If the initial profile changes sign, as it does in Fig. (1), there will be at least two shocks eventually: one moving to the left and one to the right in this reference frame, which is moving radially away from the planet at $c_{0}$.

The shock jump condition depends upon what is to be conserved. In our case, $\chi$ is approximately proportional to the density contrast $\left(\Sigma-\Sigma_{0}\right) / \Sigma_{0}$ provided that the latter is small, so mass conservation demands

$$
\left(\chi_{1}-U_{s}\right) \chi_{1}=\left(\chi_{2}-U_{s}\right) \chi_{2} \quad \Leftrightarrow \quad U_{s}=\frac{\chi_{1}+\chi_{2}}{2},
$$

where, $\chi_{1}$ and $\chi_{2}$ are the values of $\chi$ before and after the shock, and $U_{s} \equiv(d \eta / d t)_{\text {shock }}$ is the "velocity" of the shock in these variables. In physical variables, the radial velocity of the shock is the mean of the sound velocities on either side of the jump, as usual for weak shocks.

For an initial pulse that changes sign and has finite range, the final asymptotic behavior is an "N-wave," called such because the profile resembles that letter of the alphabet (Fig. 2). The unperturbed gas flows from left to right in these variables, so it first encounters the lefthand shock, followed by a rarefaction wave in which the pressure falls below its unperturbed value, and finally a jump back to the unperturbed conditions at the righthand shock. The point where $\chi=0$ (on the rarefaction wave) does not move, and the flux vanishes there, so mass conservation implies that the areas in the left and right lobes of the $\mathrm{N}$-wave are both constant. From this and the jump condition (29), it follows that the height and width of the $\mathrm{N}$-wave scale as $t^{-1 / 2}$ and $t^{+1 / 2}$, respectively (cf. Whitham 1974; Landau \& Lifshitz 1959).

We choose $x_{0}=-2 l$ as the matching point between the linear and nonlinear calculations. This is not far enough from the planet to be completely outside the excitation region: the flux in the linear wake is about $7 \%$ smaller than its asymptotic value. For $M_{p} \ll M_{1}$ one should probably use a larger value of $\left|x_{0}\right|$. In practice, the interesting values of $M_{p}$ will be not much smaller than $M_{1}$, and it is then necessary to match at a rather small $\left|x_{0}\right|$ in order to remain within the range where the linear calculations are valid.

Applying the criterion (28) to the linear wake profile at $\left|x_{0}\right|=2 l$ [see Fig. (1)], whose amplitude is $\propto M_{p}$, we find that the shock develops at $t_{\text {shock }}-t_{0} \approx 0.79\left(M_{1} / M_{p}\right)$. The constant $t_{0} \approx 1.89$ is $\propto\left|x_{0} / l\right|^{5 / 2}$ but independent of $M_{p}$. In order that subsequent formulae have a simple power-law scaling with $M_{p}$, we shall neglect $t_{0}$, but it becomes important as $M_{p}$ approaches $M_{1}$. Using the relations $(24)$ and $h \equiv c / \Omega=(2 / 3) l$, $t_{\text {shock }}$ translates to

$$
|x|_{\text {shock }} \approx 0.93\left(\frac{\gamma+1}{12 / 5} \frac{M_{p}}{M_{1}}\right)^{-2 / 5} h .
$$

This result indicates that if $M_{p} \geq M_{1}$, then the shock begins immediately and cannot be separated from the excitation region. Note that for $M_{*}=M_{\odot}$,

$$
M_{1}=\frac{2 c^{3}}{3 \Omega G} \approx 8.0\left(\frac{c}{1 \mathrm{~km} \mathrm{~s}^{-1}}\right)^{3}\left(\frac{r_{p}}{1 \mathrm{AU}}\right)^{3 / 4} M_{\oplus} .
$$




\subsection{Angular momentum flux and energy dissipation}

By summing over the contribution of each azimuthal harmonic as given by GT80, one can show that the angular-momentum flux carried by the wake in a nonselfgravitating disk is

$$
f_{J}(x)=\frac{c_{0}^{3} r_{p}}{|2 A x| \Sigma_{0}} \int_{-\pi / r_{p}}^{\pi / r_{p}}\left(\Sigma-\Sigma_{0}\right)^{2} d y .
$$

In terms of our dimensionless variables,

$$
\begin{aligned}
f_{J}(x) & =\frac{2^{3 / 2} r_{p} c_{0}^{3} \Sigma_{0}}{(\gamma+1)^{2}|2 A|} \Phi(t), \\
\text { where } \Phi(t) & \equiv \int \chi^{2}(\eta, t) d \eta .
\end{aligned}
$$

We have used the fact that the wake depends upon radius and azimuth most rapidly in the combination $\eta$, and that its slowly-varying amplitude can be thought of as a function of radius or azimuth according to the correspondence $|x| \approx \sqrt{2 l|y|}=2^{1 / 2}(5 t / 4)^{2 / 5} l$. Without shocks, the dimensionless flux $\Phi$ is strictly conserved; in fact, the integral over $\eta$ of any function of $\chi$ is conserved.

Once the shock develops, $f_{J}$ decays with distance as the angular momentum carried by the wake is transferred to the mean flow. From the scalings for the N-wave discussed above, it follows that $\Phi(t) \propto t^{-1 / 2}$ at large $t$, hence

$$
f_{J}(x) \propto|x|^{-5 / 4} \quad\left(|x| \gg|x|_{\text {shock }}\right),
$$

which was also confirmed by direct analytical $\mathrm{N}$-wave expansion of the original set of equations (22). The full evolution of $\Phi(t)$ is shown in Fig. 3.

The total energy dissipation rate associated with the absorption of the wake is [cf. eq. (4)]

$$
\begin{aligned}
\dot{e} & =2 \int_{0}^{\infty}\left[\Omega(r)-\Omega\left(r_{p}\right)\right] \frac{d f_{J}}{d x} \\
& \approx\left(\frac{4}{5}\right)^{3 / 5}\left(\frac{2}{\gamma+1}\right)^{2} c^{3} l \Sigma \int_{0}^{\infty} \Phi(t) t^{-3 / 5} d t
\end{aligned}
$$

where we have used integration by parts to transfer the radial derivative from $f_{J}$ to $\Omega(r)$. Since $t^{-3 / 5} \Phi(t) \propto t^{-11 / 10}$ at large $t$, the integral converges, but slowly; its value is $\approx 12.3$ when $M_{p}=M_{1}$.

By scaling our numerical results to other $M_{p}$ and $\gamma$, we find that the dissipation produced by a small planet is

$$
\dot{e} \approx 11 .\left(\frac{\gamma+1}{7 / 5+1}\right)^{-2 / 5} c^{3} l \Sigma\left(\frac{3 \Omega G M_{p}}{2 c^{3}}\right)^{8 / 5}
$$




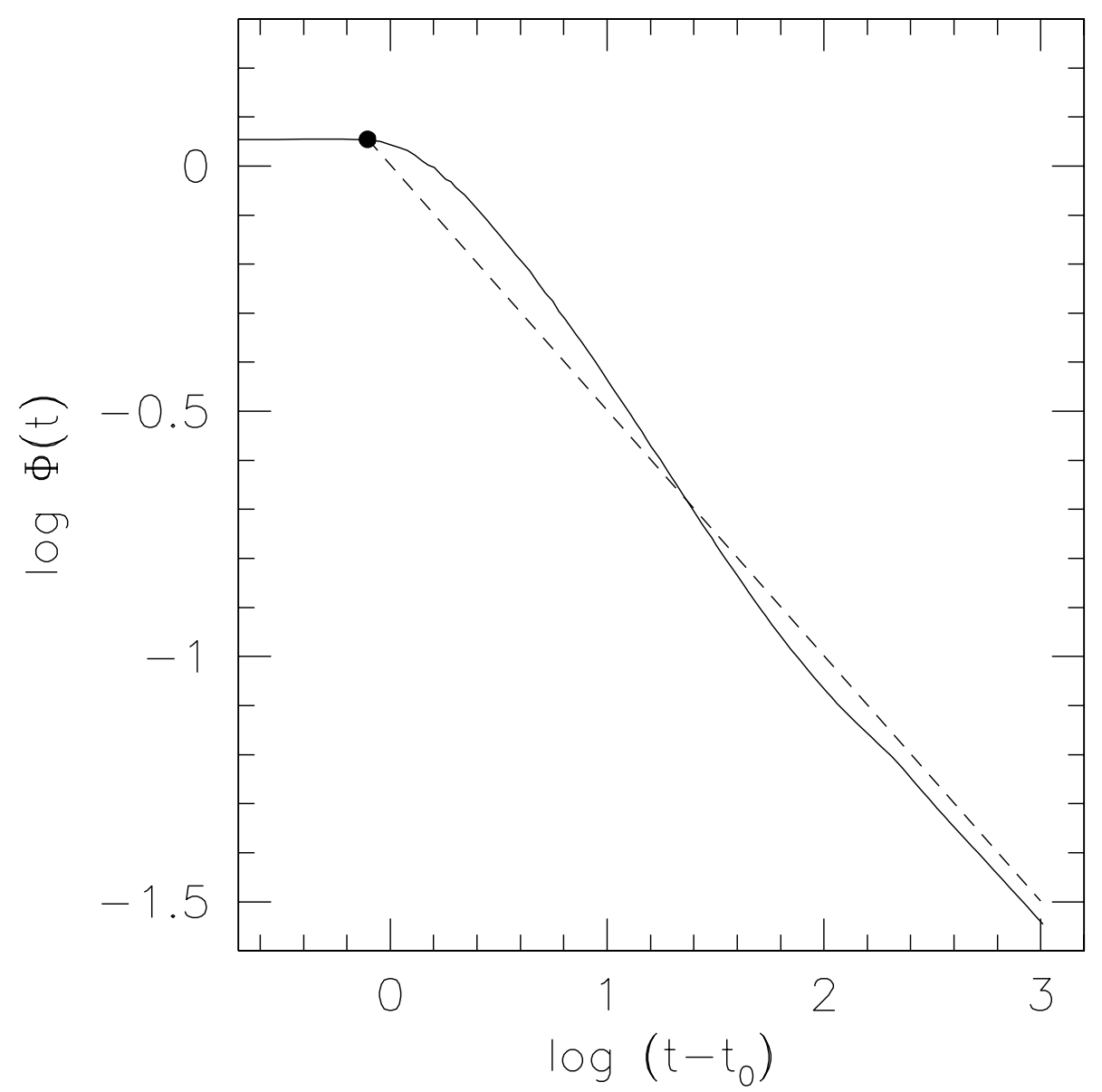

Fig. 3.- Solid line: dimensionless angular momentum flux [eq. (33)] carried by the wake versus propagation pseudotime (24), based on numerical integrations shown in Fig. 2. Dashed: Asymptotic $t^{-1 / 2}$ scaling appropriate to the $\mathrm{N}$-wave, normalized to agree with the constant preshock value $\Phi\left(t_{0}\right)$ at $t=t_{\text {shock }}($ bullet $)$. 
in a keplerian disk. From eq. (10), it now follows that effective viscosity parameter is

$$
\begin{aligned}
\alpha_{\mathrm{eff}} & =3.2\left(\frac{\gamma+1}{7 / 5+1}\right)^{-2 / 5} \frac{r^{3} c^{2} Z_{p} \Sigma}{G M_{\star}\left\langle M_{p}\right\rangle}\left\langle\left(\frac{3 \Omega G M_{p}}{2 c^{3}}\right)^{8 / 5}\right\rangle \\
& \approx 1.2 \times 10^{-4}\left(\frac{r}{\mathrm{AU}}\right)^{-1 / 5}\left(\frac{Z_{p}}{0.01}\right)\left(\frac{M_{p}}{M_{\oplus}}\right)^{3 / 5} .
\end{aligned}
$$

For simplicity we have taken all planets to have the same mass in the final line above, and we have used the MMSN model (11)-(12).

In the Abstract, we stated that shock damping is local but only weakly so. It is local in the sense that most of the contribution to $\dot{e}$ would come from a finite range of $|x| / h$ in the limit of a very thin disk, $r / h \rightarrow \infty$. But quantitatively, since protostellar disks are not extremely thin, the results of this section show that the dissipation will extend over a range comparable to the radius. Although $f_{J}(x)$ falls to half of its full value $(3)$ at $|x| \approx 2.1|x|_{\text {shock}}$, half of the dissipation occurs beyond $9.1|x|_{\text {shock }}$, and the residual contribution from distances $>|x|$ falls off as $|x|^{-1 / 4}$ when $|x| \gg|x|_{\text {shock}}$.

\section{Discussion}

We have shown that density-wave wakes excited by planets in a nonselfgravitating protostellar disk serve as an effective viscosity mechanism, provided that the waves are damped over a distance smaller than the orbital radius of the planet. The effective viscosity is proportional to the damping length, which is most likely to be limited by shocks unless the mass per planet $M_{p} \ll M_{\oplus}$. If a fixed total mass is available to form planets, then $\alpha_{\mathrm{eff}} \propto M_{p}^{3 / 5}$. Eq. (36) suggests that $\alpha_{\mathrm{eff}} \sim 10^{-4}$ in the inner solar system where $M_{p} \sim M_{\oplus}$, and $\alpha_{\text {eff }} \sim 3 \times 10^{-4}$ at $r \sim 10 A U$ if $M_{p} \sim 10 M_{\oplus}$, which is characteristic of the rock+ice cores of the giant planets, though very much less than the total mass of Jupiter or Saturn (Guillot 1999).

Our estimates for $\alpha_{\text {eff }}$ may seem optimistic because they assume that all of the rocks and ices are efficiently converted into planets. On the other hand, $\alpha_{\text {eff }}$ could be even larger if $M_{p}$ is

augmented by gas. In that case, $\alpha_{\text {eff }} \propto M_{p}^{8 / 3}$ because the mass in planets is not limited to the metals. There are two restrictions on the amount of gas we can allow for. The first is technical: we have calculated the excitation of the density waves from linear theory, which cannot be justified when $M_{p}$ exceeds the limit (31). This could be overcome by nonlinear two-dimensional numerical calculations (e.g. Korycansky \& Papaloizou 1996). The other restriction is more substantial: once $M_{p}$ is large enough to open a gap in the disk, the dissipation rate probably scales more slowly than $M_{p}^{8 / 3}$.

Several estimates exist for the minimum mass required to open a gap, $M_{\text {gap }}$ Lin \& Papaloizou (1993) and Bryden et al. (1999) argue that in a completely inviscid disk, $M_{\text {gap }} \approx M_{1}$ as given by eq. (31) because of radial gas pressure gradients; recall that at this mass, the Roche lobe (or Hill 
sphere) of the planet is $\approx h$. Ward (1997) argues for $M_{\text {gap }} \approx 0.14 \pi \Sigma h r$, or $M_{\text {gap }} \approx 0.8(r / A U)^{3 / 4} M_{\oplus}$ in the fiducial MMSN (11)-(12). This results from comparing the time to open a gap with the time to migrate across it, assuming that planetary wake damps immediately at the resonances where it is excited. Presumably $M_{\text {gap }}$ should be increased to allow for the finite damping length that we have computed, but it would still be proportional to $\Sigma$, unlike eq. (31).

$M_{\text {gap }}$ is expected to be larger in a viscous disk than in an inviscid one because the torque exerted by the planet can be balanced by a viscous torque. Bryden et al. (1999) cite

$$
\frac{M_{\text {gap }}}{M_{*}}=\frac{40 \nu}{\Omega r^{2}} \approx 40 \alpha\left(\frac{h}{r}\right)^{2},
$$

which is again independent of $\Sigma$ (if $\alpha$ is). In the MMSN, this becomes

$$
M_{\text {gap }} \approx 2 \cdot\left(\frac{\alpha}{10^{-4}}\right)\left(\frac{r}{A U}\right)^{1 / 2} M_{\oplus} .
$$

In our case, it seems likely that the shocks created by one planet will resist the opening of a gap next to an adjacent planet, so it is perhaps reasonable to use the value (36) for $\alpha$ on the righthand side above.

In the discussion to follow, we shall follow Lin \& Papaloizou (1993) and adopt (31) as a provisional value for $M_{\text {gap }}$, but clearly the question deserves further study. Then $M_{\text {gap }} \sim 45 M_{\oplus}$ at $10 \mathrm{AU}$ using eq. (12). If the planets at $10 \mathrm{AU}$ accrete enough gas to reach this limit (Saturn exceeds it by a factor $\sim 2$ ), then $\alpha_{\text {eff }} \approx 3 \times 10^{-3}$.

In short, values for $\alpha_{\text {eff }}$ in the range from $10^{-4}$ up to at least $10^{-3}$ seem plausible for the density-wave mechanism in a minimum-mass solar nebula. The predicted accretion timescale is

$$
t_{\mathrm{acc}}=\alpha^{-1}\left(\frac{r}{h}\right)^{2} \Omega^{-1} \approx 5 \times 10^{6}\left(\frac{\alpha}{10^{-3}}\right)^{-1}\left(\frac{r}{30 \mathrm{AU}}\right) \mathrm{yr},
$$

in reasonable agreement with the disk lifetimes cited in $\S 1$.

By comparing observed sizes, ages, and accretion rates of $\mathrm{T}$ Tauri disks with self-similar models, Hartmann et al. (1998) estimate $\alpha \sim 10^{-2}$, which may be too large to be explained by planetary wakes, especially if one must rely on the rocky core masses only. The strongest lower bounds on $\alpha$ assume that the disks started out much more compact than observed. In view of the wide scatter and uncertainties in the observationally derived parameters, the questionable relevance of selfsimilar models, and the theoretical uncertainties just discussed, it is unclear whether Hartmann et al.'s result is a major difficulty for us. Another concern is that planets are likely to migrate inwards with respect to the gas (Ward 1997), perhaps enhancing the viscosity of the inner disk but diminishing that of the outer parts where most of the gas probably resides. Still another is that our mechanism operates only after planet growth, which standard models place late in the lifetime of the gaseous nebula, if not afterwards (Podolak et al. 1993; Ikoma et al. 2000), whereas Hartmann et al. find larger accretion rates in younger disks on average. 
All of these objections have merit. Nevertheless, the density-wave mechanism is interesting from several points of view. First, it provides a minimum effective viscosity when other mechanisms are in abeyance. For example, MHD turbulence may dominate only during FU Orionis-like outbursts when the temperature and ionization of the disk rises (Gammie 1998); self-gravity may dominate in young, massive, cool disks (Lin \& Pringle 1987); but neither is likely to be effective in the late, thermally passive phases when it is believed that planets form. Second, calculable models for the effective viscosity are so difficult to come by that it is worthwhile to examine any plausible candidate, even if others seem more likely to dominate (but cannot be calculated). Third, the formalism offered in $\S 5$ and the Appendix for the development and damping of the shock, though only approximate, is expected to be reasonably accurate for weak shocks and simple enough to use that it may find other applications. ${ }^{1}$ Finally, there may be other nonaxisymmetric structures in protostellar disks of greater mass than planets, for example vortices (Adams \& Watkins 1995; Godon \& Livio 2000; Nauta 2000). Any such structure would create a density-wave wake.

We thank Charles Gammie, Peter Goldreich, Brad Hansen, Kristen Menou, and Scott Tremaine for helpful discussions. This work was supported by the NASA Origins Program under grant NAG58385 .

\section{REFERENCES}

Adams, F. C. \& Watkins, R. 1995, MNRAS, 317, 45

Artymowicz, P. 1993, ApJ, 419, 155.

Balbus, S.A., Hawley, J.F. \& Stone, J.M. 1996, ApJ, 467, 76

Balbus, S.A. \& Hawley, J.F. 1998, Rev. Mod. Phys., 70, 1

Blaes, O.M. \& Balbus, S.A. 1994, ApJ, 421, 163

Bryden, G., Chen, X., Lin, D. N. C., Nelson, R. P., \& Papaloizou, J. C. B. 1999, ApJ, 514, 344

Cassen, P. \& Woolum, D.S. 1996, ApJ, 472, 789

Chiang, E. I. \& Goldreich, P. 1998, ApJ, 490, 368

Dubrulle, B. 1992, A\&A, 266, 592

Gammie, C.F. 1996, ApJ, 457, 355

Gammie, C.F. 1998, in Asp. Conf. Ser. 160, Astrophysical Discs, ed. J. A. Sellwood \& J. Goodman (San Francisco: ASP), 122

\footnotetext{
${ }^{1}$ Burger's equation can be solved analytically given a closed form for the initial wave profile (cf. Whitham 1974).
} 
Gammie, C.F. 2000, in preparation.

Godon, P. \& Livio, M. 2000, ApJ, 537, 396

Goldreich, P. \& Nicholson, P.D. 1989, ApJ, 342, 1075

Goldreich, P. \& Tremaine, S. 1978, ApJ, 222, 850 (GT78).

Goldreich, P. \& Tremaine, S. 1980, ApJ, 241, 425 (GT80).

Gonzalez, G. 1997, MNRAS, 285, 403

Gonzalez, G., Laws, C., Tyagi, S., \& Reddy, B. E. 2000, astro-ph/0010197

Hartmann, L., Calvet, N., Gullbring, E., \& D’Alesio, P. 1998, ApJ, 495, 385

Hartmann, L., Kenyon, S., \& Hartigan, P. 1993, in Protostars and Planets III, ed. E. H. Levy \& J. I. Lunine (Tucson:Univ. Arizona Press), 497

Hayashi, C. 1981, Progr. Theor. Phys. Suppl., 70, 35

Hawley, J.F. \& Stone, J.M. 1998, ApJ, 501, 758

Ikoma, M., Nakazawa, K., Emori, H. 2000, ApJ, 537, 1013

Jin, L., 1996, ApJ, 457, 798

Korycansky, D. G. \& Papaloizou, J. C. B. 1996, ApJS, 105, 181

Landau, L. D \& Lifshitz, E. M. 1959, Fluid Mechanics, (Oxford: Pergamon).

Larson, R.B. 1989, In: The Formation and Evolution of Planetary Systems, p.31, eds. Weaver, H.A. \& Danly, L., Cambridge University Press, Cambridge

Larson, R.B. 1990, MNRAS, 243, 588

Lin, D.N.C. \& Papaloizou, J. 1980, MNRAS, 191, 37

Lin, D.N.C. \& Papaloizou, J. 1993, in Protostars and Planets III, ed. E. H. Levy \& J. I. Lunine (Tucson:Univ. Arizona Press), 749

Lin, D. N. C. \& Pringle, J. E. 1987, MNRAS, 225, 607

Lubow, S.H. \& Ogilvie, G.I. 1998, ApJ, 504, 983

Lynden-Bell, D. \& Pringle, J. E. 1974, MNRAS, 168, 603

Marcy, G. W., Cochran, W. D., Mayor, M., 2000, in Protostars and Planets IV, ed. V. Manning, A. Boss, \& S. Russell (Tucson: Univ. Arizona Press), 1285 
Mizuno, H., Nakazawa, K., \& Hiyashi, C. 1978, Prog. Theor. Phys. 60, 699

Nauta, M. D. 2000, Two-Dimensional Vortices and Accretion Disks, Ph.D. Thesis, Utrecht

Ogilvie, G.I. \& Lubow, S.H. 1999, ApJ, 515, 767

Osterloh, M. \& Beckwith, S.V.W. 1995, ApJ, 439, 288.

Podolak, M., Hubbard, W. B., \& Pollack, J. B. 1993, in Protostars and Planets III, ed. E. H. Levy \& J. I. Lunine (Tucson:Univ. Arizona Press), 1109

Podosek, F. A. \& Cassen, P. 1994, Meteoritics, 29, 6

Pringle, J.E. 1981, ARA\&Ap, 19, 137

Guillot, T. 1999, Science, 286, 72.

Ryu, D. \& Goodman, J. 1992, ApJ, 388, 438

Santos, N. C., Israelian, G., \& Mayor, M. 2000, astro-ph/0009182

Shakura, N. I. \& Sunyaev, R. A. 1973, A\&A, 24, 337

Spruit, H.C. 1987, 184, 173

Stone, J.M. \& Balbus, S.A. 1996, ApJ, 464, 364

Strom, K. M., Strom, S. E., Edwards, S., Cabrit, S., \& Skrutskie, M. F., 1989, AJ, 9, 1451.

Takeuchi, T., Miyama, S.M., \& Lin, D.N.C. 1996, ApJ,460, 832

Toomre, A. 1964 ApJ, 139, 1217

Ward, W.R. 1997, Icarus, 126, 261.

Wardle, M. 1999, MNRAS, 307, 849

van Leer, B. 1977, J. Comp. Phys., 23, 276

Whitham, G. B. 1974, Linear and Nonlinear Waves, (New York: Wiley).

Zahn, J.-P. 1991, in Structure and Emission Properties of Accretion Disks, ed. C.Bertout et al. (Gif sur Yvette:Edi. Frontires), 87

This preprint was prepared with the AAS LATEX macros v5.0. 


\section{A. Reduction to Burger's equation}

At $|x| \gg l$, eqs. (22) are hyperbolic, as usual for supersonic steady flows (Landau \& Lifshitz 1959): that is, they describe an initial-value problem. It is convenient to regard $y$ as the timelike variable. The three characteristic "velocities" are

$$
\left(\frac{d x}{d y}\right)_{0,+,-}=\frac{u}{2 A x+v}, \frac{u(2 A x+v) \pm c \sqrt{(2 A x+v)^{2}+u^{2}-c^{2}}}{(2 A x+v)^{2}-c^{2}} .
$$

Eqs. (22) simplify for $|x| \gg l$, where a linearized WKB treatment suggests that $\partial_{y} \ll \partial_{x}$ and $v \ll u$. Dropping $v$ entirely, and $\partial_{y}$ except in the combination $2 A x \partial_{y}$, results in

$$
\begin{aligned}
\partial_{y} u+u \partial_{\xi} u+\frac{c^{2}(\Sigma)}{\Sigma} \partial_{\xi} \Sigma & =0 \\
\partial_{y} \Sigma+u \partial_{\xi} \Sigma+\Sigma \partial_{\xi} u & =0 .
\end{aligned}
$$

We have replaced $x$ with $\xi \equiv A x^{2}$ so that the equations are autonomous, i.e., the independent variables do not appear explicitly.

The system (A1) is formally identical to one-dimensional isentropic gas dynamics and can be solved by the same methods(Landau \& Lifshitz 1959). It has characteristics $C_{ \pm}$and Riemann invariants $R_{ \pm}$

$$
C_{ \pm}:\left(\frac{d \xi}{d y}\right)_{ \pm}= \pm c+u, \quad R_{ \pm} \equiv u \pm \frac{2 c}{\gamma-1}
$$

The $C_{+}$characteristics propagate toward the planet from the unperturbed disk, where $u=0$ and $c=c_{0}$. Therefore $R_{+}=2 c_{0} /(\gamma-1)$ everywhere, and $u=2\left(c_{0}-c\right) /(\gamma+1) .^{2}$ Eqs. (A1) then reduce to a single first-order equation,

$$
\begin{aligned}
0 & =\partial_{y} \psi-(1+\psi) c_{0} \partial_{\xi} \psi \\
\psi(y, \xi) & \equiv \frac{\gamma+1}{\gamma-1} \frac{c-c_{0}}{c_{0}} \approx \frac{\gamma+1}{2} \frac{\Sigma-\Sigma_{0}}{\Sigma_{0}} \quad \text { if } \psi \ll 1,
\end{aligned}
$$

which is the well-known inviscid Burger's equation: the simplest nonlinear equation capable of displaying a shock (Whitham 1974).

Unfortunately, the analysis above is oversimple. In the limit of an infinitesimal disturbance, eqs. (A1) predict that the wave propagates at constant amplitude. But a linear WKB treatment of eqs. (22) shows that the amplitude of such a wave grows in proportion to $|x|^{1 / 2} \propto|\xi|^{1 / 4}$ as a consequence of conservation of angular momentum flux. To recover this behavior, the larger subdominant terms involving $v$ that were omitted in passing from eqs. (22) to (A1) must be reinstated. The equations for the $C_{ \pm}$characteristics become

$$
\partial_{ \pm} R_{ \pm} \approx \frac{1}{2 A x}\left(2 \Omega v \mp c \partial_{y} v\right) \equiv S_{ \pm}
$$

\footnotetext{
${ }^{2}$ We take $x<0$ and $y>0$, which describes the wake interior to the orbit of the planet. The discussion that follows is equally valid for $x>0$ and $y<0$ if one interchanges the roles of $C_{+}$and $C_{-}$
} 
where $R_{ \pm}$have the same meaning as before, and we have introduced the abbreviations $\partial_{ \pm} \equiv$ $\partial_{y}+(u \pm c) \partial_{\xi}$ for the derivatives along $C_{ \pm}$. The source terms $S_{ \pm}$are smaller than individual terms in $R_{ \pm}$by $O\left(\xi^{-1}\right)$ but nonzero, so that the $R_{ \pm}$are not invariant. Yet $R_{+}$is still well approximated by its undisturbed value $2 c_{0} /(\gamma-1)$ : First of all $R_{+}$is truly undisturbed until the incoming $C_{+}$ characteristic encounters the leading edge of the wake, which propagates in the opposite direction on a bundle of $C_{-}$characteristics of roughly constant width $\Delta \xi$. Then, since the encounter lasts a "time" $\Delta y \sim \Delta \xi / 2 c$, the maximum change in $R_{+}$relative to its undisturbed value is $O(\Delta \xi / \xi) \ll 1$, which may be neglected at large $\xi$.

The source term $S_{-}$cannot be neglected because it "travels with" the wake so that its effects accumulate. We solve for $S_{-}$using the second of eqs. (22), which, to an adequate approximation, reads

$$
\partial_{y} v \approx-\frac{1}{2 A x}\left(2 B u+\frac{c^{2}}{\Sigma} \partial_{y} \Sigma\right)
$$

Only those parts of $S_{-}$that are in phase with $u$ or $\Sigma-\Sigma_{0}$ are important for the growth of the amplitude; the out-of-phase parts make a slight but negligible change to the characteristic velocity. The operator $\partial_{y}$ changes the phase by $90^{\circ}$, so from the equation above,

$$
-\frac{1}{2 A x}\left[c \partial_{y} v+2 \Omega v\right]_{\text {in phase }}=-\frac{1}{2 A \xi}\left(2 B c u+2 \Omega \frac{c^{2}-c_{0}^{2}}{\gamma-1}\right)
$$

We approximate $c$ by $c_{0}$ except in the difference $c-c_{0}$ and invoke the approximate constancy of $R_{+}$to write $u \approx 2\left(c_{0}-c\right) /(\gamma-1)$. Finally we write $\psi \equiv(\gamma+1)\left(c-c_{0}\right) /(\gamma-1) c_{0}$ as before, and so obtain the corrected $C_{-}$equation in the form

$$
\partial_{y} \psi-(1+\psi) c_{0} \partial_{\xi} \psi=-\frac{c_{0}}{4 \xi} \psi
$$

As expected, the general solution of the linearized form of eq. (A4) is $\psi=|\xi|^{1 / 4} f\left(\xi+c_{0} y\right)$, where $f$ is an arbitrary function. The full equation incorporates all three processes that dominate propagation of the wake:

(i) increasing radial wavenumber with distance from the planet $\left(\partial_{x}=2 A x \partial_{\xi}\right)$;

(ii) increasing amplitude due to conservation of angular-momentum flux;

(iii) nonlinear steepening.

Fortunately, eq. (A4) can be transformed back into Burger's equation by changing the independent and dependent variables. First let $\xi \rightarrow c_{0} l(\eta-y / l)$ (so $\eta$ is dimensionless):

$$
l \partial_{y} \psi-\psi \partial_{\eta} \psi=\frac{l}{4 y} \psi
$$

Since the wake is confined to $|\eta| \ll y / l$, we have approximated $y-\eta l$ by $y$ on the righthand side. Next, let $\psi=(y / l)^{1 / 4} \chi$ to absorb the source term:

$$
l \partial_{y} \chi-(y / l)^{1 / 4} \chi \partial_{\eta} \chi=0 .
$$

Finally, introduce a new dimensionless "time" (or azimuth) variable $t$ as in eq. (24), so that the dimensionless equation of motion becomes (23). 